Pure \& Appl. Chem., Vol. 57, No. 8, pp. 1091-1102, 1985.

Printed in Great Britain.

(C) 1985 IUPAC

\title{
Theories of transport properties using the Onsager treatment
}

\author{
Jean-Claude Justice \\ Laboratoire d'Electrochimie, Université Pierre et Marie Curie, 4 Place Jussieu, 75230 Paris Cedex 05, France.
}

\begin{abstract}
The fundamental equations of the Onsager approach of transport properties in linear response are summarized. From a reformulation of the part of the ion pair distribution function which is perturbed by the ionic velocities, an echo formulation for tracer diffusion is derived which can be compared with former similar results obtained for conductance. It clearly appears that these two different transport processes are in agreement with the original Bjerrum formulation of ionic association for excess thermodynamic properties at equilibrium which can be derived from the Mayer activity expansion.

It is finally shown how the Pikal equation for chemical diffusion can be reformulated to reach a final expression also in complete agreement with a Bjerrum formulation. The improvement in overall efficiency of these echo formulations for ions of higher charges and for solvents of lower dielectric constants is emphasized as well as their evident ability to be easily extended to more realistic models for ionic interactions than the restricted primitive model.
\end{abstract}

\section{INTRODUCTION}

The literature abounds in contributions to the study of the properties of electrolyte solutions which make use of the approach originated by Debye (ref. 1), and Falkenhagen (ref. 2,3), and formally developed by Onsager and Fuoss (ref. 4). These contributions deal with the theories of activity coefficients and transport properties. They constitute a number of attempts to extend the initial results to a wider range of uses: from symmetrical electrolytes to those of any types, from solutions of a single electrolyte to mixtures, from very low to moderate concentrations, from solvents with a high dielectric constant to lower ones and possibly to combinations of these less and less restrictive conditions of application. One of these attempts, however, cannot immediately be connected, at least at first sight, with the Onsager approach. Rather, it seems merely to constitute an empirical attempt to graft a chemical insight onto a physical treatment. Such is the case of the well-known Bjerrum extension (ref. 5), which makes use of the chemical concept of association, quantitatively treated by a mass-action law formulation, the equilibrium constant of which being the only point through which the linkage with the Debye treatment occurs. This chemical extension was also the object of suggestions for amendments, most of which have probably brought more confusion, rather than clearer insight, into the understanding of the processes involved at the molecular level.

Thus it seems not unimportant that experimenters, who are those most directly interested in making good use of a theoretical equation, should be offered some sort of a general and hopefully simple survey so that they can more easily make their own judgement on several fundamental points such as:

A- How deeply is the Onsager approach embedded in the rigorous treatment of statistical mechanics?

B- Where exactly do the various approximations used at different steps of the mathematical development lie and what are their physical meanings?

C- Is there one way of comparing the various theoretical equations available and deciding eventually if one of them might exhibit a significant advantage over the others?

D- Since the association models have proved so efficient, whatever kinds of theoretical criticism may have been levelled at this concept, is there one better than the others and how can it be related firmly to the fundamental bases of statistical mechanics? 
Positive answers to these questions are now at hand. Some can already be found in the literature. Unfortunately, they are often formulated in the precise but obscure jargon of specialists and embedded in an intricate mathematical formalism which is not obviously clear to the not necessarily well-armed though curious experimenter in the field. The intention of this paper is to provide some help in circumventing this difficulty. It has been carried out in the hope of being useful to those experimenters dealing with electrolyte solutions, who probably often felt discouraged, as the author also often did, by the apparent complexity and seeming contradictions found on reading the literature.

We shall make use of a symbolism which is a generalisation of that originally introduced by Falkenhagen and which greatly facilitates the direct analysis and understanding of the exact physical content of the different relations using various timeaveraged quantities. In superscript is found the information concerning the species and the location to which the quantity belongs. In subscript is found the information concerning the restricting conditions to be fulfilled during the averaging time regarding the same quantity. For instance, $g_{i P, j Q}^{l S}$ is the probability density for finding an ion of type $l$ at $S$ while two ions of type $i$ and $j$ are present at $P$ and $Q$ respectively. The extension of this formalism to any other mean quantity such as mean velocity vectors, mean interaction forces ...etc, is straightforward.

It is then possible to summarize the Onsager approach for the treatment of the physico-chemical properties of the electrolyte solutions in a general set of equations (cf. table 1) which can be later specialized to the particular conditions defining the macroscopic properties to be studied ( equilibrium excess thermodynamic properties, static and frequency-dependent conductance, self- or tracer-diffusion, mutual or chemical diffusion ...etc).

\section{TABLE 1}

Summary of the Four Basic Sets of Hierarchy Equations in the Onsager Treatment of the Physico-Chemical Properties of Electrolyte Solutions

1. Total Velocities

$$
\begin{gathered}
\mathbf{v}^{i P}=\mathbf{v} e^{i P}+\omega_{i}\left(\mathbf{K}_{i}+\mathbf{F}^{i P}\right) \\
\mathbf{v}_{j Q}^{i P}=\mathbf{v} e_{j Q}^{i P}+\omega_{i}\left(\mathbf{K}_{i}+\mathbf{F}_{j Q}^{i P}\right) \\
\ldots \ldots \ldots \text { etc } \ldots \ldots \ldots \ldots
\end{gathered}
$$

2. Internal Forces

$$
\begin{gathered}
\mathbf{F}^{i P}=-k_{B} T \nabla_{P} \ln g^{i P}-\sum_{l} n_{l} \int \nabla_{P} U(i P, l S) g_{i P}^{l s} d V_{S} \\
\mathbf{F}_{j Q}^{i P}=-k_{B} T \nabla_{P} \ln g_{j Q}^{i P}-\nabla_{P} U(i P, j Q)-\sum_{l} n_{l} \int \nabla_{P} U(i P, l S) g_{i P, j Q}^{l S} d V_{S} \\
\mathbf{F}_{j Q, k R}^{i P}=-k_{B} T \nabla_{P} \ln g_{j Q . k R}^{i P}-\nabla_{P} U(i P, j Q)-\nabla_{P} U(i P, k R)-\sum_{l} n_{l} \int \nabla_{P} U(i P, l S) g_{i P, j Q, k R}^{l S} d V_{S}
\end{gathered}
$$

3. Electrophoretic Velocities

$$
\begin{gathered}
\mathbf{v} e^{i P}=\sum_{l} n_{l} \int \vec{\chi}\left(P, S ; \omega_{i}, \omega_{l}\right)\left(\mathbf{K}_{l}+\mathbf{F}_{i P}^{l S}\right) g_{i P}^{l S} d V S \\
\mathbf{v} e_{j Q}^{i P}=\sum_{l} n_{l} \int \vec{\chi}\left(P, S ; \omega_{i}, \omega_{l}\right)\left(\mathbf{K}_{l}+\mathbf{F}_{i P, j Q}^{l S}\right) g_{i P, j Q}^{l S} d V_{S}+\vec{\chi}\left(P, S, \omega_{i}, \omega_{j}\right)\left(\mathbf{K}_{j}+\mathbf{F}_{i P}^{j Q}\right)
\end{gathered}
$$

4. Mass Conservation

$$
\begin{gathered}
-\frac{\partial}{\partial t} g^{i P}=\nabla_{P} g^{i P} \mathbf{v}^{i P} \\
-\frac{\partial}{\partial t} g_{j Q}^{i P}=\nabla_{P} g_{j Q}^{i P} \mathbf{v}_{j Q}^{i P}+\nabla_{Q} g_{i P}^{j Q} \mathbf{v}_{i P}^{j Q}=-\frac{\partial}{\partial t} g_{i P}^{j Q} \\
\ldots \ldots \ldots \ldots \ldots \ldots \ldots \ldots \text { etc } \ldots \ldots \ldots \ldots \ldots \ldots \ldots \ldots
\end{gathered}
$$


First, it must be specified that in the Onsager treatment, the solvent is considered as a dielectric and hydrodynamic continuum characterized by a dielectric constant $\epsilon$ and a viscosity $\eta$. This has been proved by McMillan and Mayer to be a sound basis for the theoretical treatment of equilibrium excess properties (that is, excluding transfer or direct ion-solvent properties) provided that a realistic enough solute-solute interaction model is used to supplement the consequences of the ignorance of the molecular nature of the solvent.

The most commonly used interaction model is also the most simple to handle in the mathematical derivations. It is called the restricted primitive model (RPM). It makes use of the following potential energy of interaction $U_{i j}(r)$ between two ions of type $i$ and $j$

$$
U_{i j}(r)= \begin{cases}\infty, & \text { if } r<a \\ e_{i} e_{j} / \epsilon r, & \text { otherwise. }\end{cases}
$$

When displayed in the formal presentation of the fundamental equations, the notation of the function $U_{i j}(r)$ will be replaced by $U(i P, j Q)$ in order to be consistent with the symbolism used for the mean quantities mentioned in the same equation, which explicitly refer to the locations $P$ and $Q$ for instance.

More realistic models can be used which will be described later, the Onsager treatment being not strictly bound to the RPM. However this model, because of the relative simplicity it allows in the mathematical derivations, is quite useful in reaching many interesting and general conclusions.

\section{THE BASIC EQUATIONS}

\section{2-1. The mean one-particle velocity vector $\mathrm{v}^{i P}$}

This quantity is defined by eq.(1) in table 1 . It expresses the mean velocity vector of an ion of type $i$ located at $P$, induced by an external force $\mathbf{K}_{i}$ to which must be added an internal force $\mathbf{F}^{i P}$ exerted by all the other ions of the solution. The product of these forces with the intrinsic mobility $\omega_{i}$ of the ion in the pure solvent gives a first vector contribution for the velocity of an ion of type $i$ with respect to the solvent at $P$, to which must be added a contribution $v e^{i P}$ called the electrophoretic velocity of the ion of type $i$ at $P$, which is due to an eventual hydrodynamic flow of the medium at $P$.

As a matter of fact, the mean one-particle velocities are the quantities which are measured as transport properties, under more or less disguised transformations, according to the technique used and experimental conditions imposed. The transposition from $\mathbf{v}^{i P}$ to the ionic conductance $\lambda^{i}$, and to the self-diffusion coefficient $D_{a}^{i}$ is only a matter of performing the following vectorial operations.

$$
\begin{aligned}
& \mathbf{v}^{i P}=\mathbf{X} \lambda^{i} / F z_{i} \\
& \mathbf{v}^{i P}=-D_{a}^{i} \nabla_{P} \ln g^{i P}
\end{aligned}
$$

where $\mathrm{X}$ is the externally applied electrical field, $\left(\mathbf{K}_{i}=z_{i} e \mathrm{X}\right), F$ the Faraday constant and $e$ the proton charge. The case of the chemical diffusion coefficient is somewhat more complicated and will be briefly summarized in section 6-3.

\section{2-2. The mean one-particle internal force $\mathrm{F}^{i P}$}

As shown in eq.(3), it contains two contributions:

A- A fictitious force, $-k_{B} T \nabla_{P} \ln g^{i P}$, called diffusion force which is non-zero if the local concentration of the concerned ion is not uniform at $P$. This term corresponds to the driving force in the problem of diffusion.

B- A contribution which is due to the direct interaction force, $-\nabla_{P} U(i P, l S)$, exerted by each of the other ions of the solution whose local mean concentration in the volume element $d V_{S}$ is equal to $n_{l} g_{i P}^{l S}$ since an ion $i$ is present at $P$. Let us note here that $n_{l}$ is a constant quantity defined by the ratio $N_{l} / V$, where $N_{l}$ is the total number of ions of type $l$ originally introduced in the whole volume $V$ offered to the solution.

Thus, the evaluation of the internal force $\mathrm{F}^{i P}$ necessitates the knowledge of both distribution functions $g^{i P}$ and $g_{i P}^{l S}$. The oneparticle distribution function is generally a known quantity which is a constant equal to unity, if the solution is homogeneous, or which varies, in diffusion experiments, like the ratio of the locally-controlled concentration, $n^{l s}=n_{l} g^{l s}$, to the overall concentration $n_{l}$ defined above. The case of the two-ion distribution functions is different since these quantities are unknown We shall see that all the physico-chemical problems of solutions always ultimately reduce to the evaluation of pair distribution functions. 


\section{2-8. The mean one-particle electrophoretic velocity vector $\mathbf{v} e^{i P}$}

When an ion is moving with a time-averaged velocity vector which is not zero, its corresponding momentum is transmitted entirely to the supporting medium ( since an equilibrium is reached between the driving force acting on the ions and the hydrodynamic reaction of the medium ). As a consequence, any ionic motion causes a local hydrodynamic flow of the medium and conversely, any ion immersed in the medium will experience a hydrodynamic force from the medium which will "push" it with a velocity $\mathbf{v} e^{i P}$ in the direction of the local flow. The evaluation of this velocity component $\mathrm{v} e^{i P}$ is indeed a complicated problem since it involves a number of intermediate quantities which participate at different levels in a double ion-solvent and solvent-ion hydrodynamic coupling which, in addition, is acting in conjunction with the direct ion-ion coupling.

Fortunately this problem has received with eq.(6) a remarkably simple formulation which can be given the following interpretation. Let us consider a volume element $d V_{S}$, centered at point $S$ and an ion of type $i$ located at $P$. All the ions contained in $d V s$ exert on this volume element a driving force

$$
d \mathbf{F}^{s}=\sum_{l} n_{l}\left(\mathbf{K}_{l}+\mathbf{F}_{i P}^{l S}\right) g_{i P}^{l S} d V_{s}
$$

The laws of hydrodynamics tell us that this force, exerted on $d V_{s}$ by particles of mobility $\omega_{l}$, induces in the fluid a flow whose net action is to increase the velocity of a particle $i$ at $P$ of mobility $\omega_{i}$ by the amount $\overrightarrow{\vec{\chi}}\left(P, S, \omega_{i}, \omega_{l}\right) d \mathrm{~F}^{s}$ where $\overrightarrow{\vec{\chi}}$ is a tensor operator given in appendix $\mathrm{A}$.

As a consequence, it gives for the electrophoretic velocity increment

$$
d \mathbf{v} e^{i P}=\sum_{l} n_{l} \vec{\chi}\left(P, S, \omega_{i}, \omega_{l}\right)\left(\mathbf{K}_{l}+\mathbf{F}_{i P}^{l S}\right) g_{i P}^{l S} d V_{S}
$$

whose integration over the whole space leads to the final formulation of the electrophoretic velocity vector $v e^{i P}$ given by eq.(6) of table 1 .

\section{2-4. The continuity equation for the two-particle distribution functions.}

When the solute particles are in motion, depending on their velocities, their distribution functions may be affected or not. In the absence of any local source or trap of particles, eq.(8) expresses the variation with time and space of the local concentration as a function of the local velocity of the species considered. Eq.(8) merely expresses the mass conservation. A corresponding relation holds between the pair-distribution functions and the two-particle mean velocity vectors as shown in eq.(9). This equation is important since its solution, using appropriate boundary conditions, allows the evaluation of the two-particle distribution functions which are key functions for the evaluations of $\mathrm{F}^{i P}, \mathrm{v} e^{i P}$ and thus of $\mathrm{v}^{i P}$. A careful analysis shows that the continuity eq.(9) allows only the evaluation of that part of the pair distribution function $g_{j Q}^{\prime i P}$ which depends on the ion motion and which has been shown (ref. 6), to be expressible according to the following general formulation in the case of linear irreversibility:

$$
g_{j Q}^{i P}=Y_{i j}(r) \cos \theta \frac{\left(\mathbf{v}^{i P}-\mathbf{v}^{j Q}\right) \nabla x}{k_{B} T\left(\omega_{i}+\omega_{j}\right)}
$$

Here, $r$ is the distance $P Q ; \nabla x$ is the unit vector in the direction of the driving force; $\theta$ is the angle $(P Q, \nabla x) ; k_{B}$ is the Boltzmann constant and $T$ the Kelvin temperarure.

The solution for $Y_{i j}(r)$ of course depends on the value which is introduced in the continuity equation for the other part which is independent of the ionic motion and which is supposed to be known. In conductance that part identifies with the equilibrium pair distribution function commonly denoted $g_{j Q}^{i P}$, but in ionic diffusion and particularly in chemical diffusion the situation is different since the distribution functions are already perturbed by the imposed gradient of concentration. As a consequence one can write

$$
g_{j Q}^{i P}=g_{j Q}^{i P}+g_{j Q}^{\nabla i P}+g_{j Q}^{i P}
$$

where $g_{j Q}^{\nabla i p}$ stands for the perturbation part due to the local concentration gradient which exists in the case of diffusion.

\section{2-5. The hierarchies of the transport equations.}

Along the different steps in the presentation of the successive problems to be attacked in the Onsager treatment, from the definition of $\mathbf{v}^{i P}$ to the evaluation of $g_{i P}^{l}$, one observes a progression in the order of complexity concerning the restrictive conditions in the time-averaging of the quantities concerned. This ended in a closed loop, since from considerations about $\mathbf{v}^{i P}$, which is the final goal of the theoretical problem, one now needs an evaluation of all the $v_{j Q}^{i P}$ vectors for all possible couples of ions. It is not difficult to develop a systematic formalism which in turn will proceed, as formerly, from $v_{j Q}^{i P}$ upward by a succession of loops, containing the same sequence of equations. Indeed, each equation presented in section 2 can easily be generalized by climbing, each time, one step in the number of conditions (the couples of subscripts in the symbolism used here) 
Each quantity leads to a whole set of similar equations which thus constitute a hierarchy. In table 1 , only the first equations of each hierarchy are presented and it would be a trivial problem to proceed further up. This is, however, unnecessary since an approximation is commonly used which will stop this practically endless process of forward flight at the level of the second order in each hierarchy.

\section{THE KIRKWOOD AND THE DEBYE APPROXIMATIONS}

It will first be assumed that the three-particle conditional distribution function can be expressed as the product

$$
g_{i P, j Q}^{l S}=g_{i P}^{l S} \times g_{j Q}^{l S}
$$

which assumes that the probability of a three-ion configuration is pair-wise uncorrelated.

This indeed is not a very drastic restriction. It is always observed if the total potential energy of the system is pair-wise additive. Unfortunately, this first approximation is not sufficient to make the mathematics much easier to handle and a second approximation must be introduced. The most simple one consists in neglecting the last term in

$$
g_{i P}^{l S} g_{j Q}^{l S} \equiv\left(1+h_{i P}^{l S}\right)\left(1+h_{j Q}^{l S}\right)=1+h_{i P}^{l S}+h_{j Q}^{l S}+h_{i P}^{l S} h_{j Q}^{l S}
$$

Neglecting the cross-product term $h_{i P}^{l S} h_{j Q}^{l S}$ of the correlation functions does indeed simplify the mathematical problem substantially, but it also limits the range of validity of the final theoretical equation to more dilute concentrations. Specializing now in the space-range where the interaction functions are purely coulombic and substituting the above approximation in eq.(4) leads to a straightforward derivation of the Poisson-Boltzmann equation

$$
\Delta \psi_{j Q}^{P}=-\frac{4 \pi}{\epsilon} \sum_{k} n_{k} e_{k} g_{j Q}^{k R}=-\frac{4 \pi}{\epsilon} \rho_{j Q}^{P}
$$

where $\psi_{j Q}^{P}$ is the mean electrostatic potential at $P$ when an ion of type $j$ is present at $Q$ and where $\rho_{j Q}^{P}$ is the mean electrical charge density at $P$ in the same restrictive conditions. One can but be struck by the fact that this result is in strict conformity with the classical law of Poisson in electrostatics, which is itself a direct consequence of the Maxwell equations and such was indeed the leading idea of Debye when he used it as a starting point. However, the Poisson law is applicable only to "real" , not to "average", configurations. At a given time, given a real configuration, it is exact, but for the average potential versus the average ionic density, it is not. We know now what is the approximation involved in the daring proposition of Debye and that in neglecting the cross-product term in eq.(16), one not only seriously reduces the range of application of the theory by neglecting triplet effects of order 2 in concentration but one also loses the observation of the linear superposition of the potentials. This last point was of course realized by Debye, but it happened that linearizing the PB equation, in a third approximation, led to the well-known result

$$
\Delta \psi=\kappa^{2} \psi
$$

which was no longer affected by this deffect. This does not mean however that eq.(18) is better than eq.(17). The opposite is true.

\section{THE FUOSS-ONSAGER CONTINUITY EQUATION}

It is possible to substitute succesively all eqs.(2-7) in eq.(9), to obtain ultimately an integral-differential equation which contains only distribution functions of second to fourth order as unknown quantities. The result identifies, then, with the continuity equation derived by Ebeling (ref. 7), after some approximations, from the Liouville equation which expresses the dynamic evolution of a given distribution of interacting ion and solvent particles, in presence or not of an externally-applied perturbing force.

If one now introduces in the Ebeling equation, the Kirkwood approximations described in the previous section, one obtains a continuity equation which is a function of pair-distributions only,

$$
-\frac{1}{k_{B} T\left(\omega_{i}+\omega_{j}\right)} \frac{\partial}{\partial t} g_{j i}^{\prime}(\mathbf{r})=\Delta g_{j i}^{\prime}(\mathbf{r})+\sum_{l=1}^{2} S_{l}+\sum_{l=1}^{9} T_{l}
$$

where $g_{j i}^{\prime}(\mathbf{r})$ stands for $g_{j Q}^{\prime i P}$ which was defined in eq.(13), and where $S_{l}$ and $T_{l}$ terms are to be found elsewhere (ref. 8). The $S_{l}$ terms contribute to the limiting law and the $T_{l}$ terms to higher order terms only. It is then easy to identify each of the $S_{l}$ and $T_{l}$ terms with those of the Fuoss-Onsager continuity equation, except for some which are simply absent due to further approximations or omissions in the phenomenological approach used. It is also straightforward to compare the various treatments of Pitts, Fuoss, Falkenhagen, Murphy and Cohen, Carman, Lee and Wheaton, Quint and Viallard, and Chen. This comparison is to be found in table 2 of (ref. 8 ). 


\section{THE EQUILIBRIUM DISTRIBUTION FUNCTIONS}

These quantities are of paramount importance and they must be introduced as analytically known functions in the various equations of the Onsager treatment. Use is often made of the classical Debye-Huckel (DH) truncated expansion functions, but in that case one knows that even the linear contributions in concentration in all excess quantities cannot be exact. Another use is the so-called $\mathrm{DH}$-exponential ( $\mathrm{DHX}$ ) equation

$$
\stackrel{\circ}{g}_{i j}=\exp \left(-\frac{z_{i} z_{j}}{\left|z_{i} z_{j}\right|} \frac{2 q}{r} e^{-\kappa r} \frac{e^{\kappa a}}{1+\kappa a}\right)
$$

or simply the Meeron equation

$$
\stackrel{\circ}{i j}_{i j}=\exp \left(-\frac{z_{i} z_{j}}{\left|z_{i} z_{j}\right|} \frac{2 q}{r} e^{-\kappa r}\right)
$$

with $q=z^{2} e^{2} / \epsilon k_{B} T$, commonly called the Bjerrum distance. It has been shown (ref. 9,10), that with these two equations the linear contributions for the excess thermodynamic quantities at equilibrium can be derived exactly and one may legitimately presume that such is also the case for the transport excess functions.

One can still make one step further in the improvement regarding the analytical expression for $\stackrel{g}{i j}_{i j}$. It can be shown (ref. 11), from considerations dealing with the Mayer activity expansion that, given an exact formulation for the mean activity coefficient of a RPM electrolyte $f_{ \pm}(a, c)$, an equivalent formulation is

$$
\begin{gathered}
f_{ \pm}(a, c)=\gamma f_{ \pm}(R, c \gamma) \\
\frac{1-\gamma}{\gamma^{2} c f_{ \pm}^{2}(R, c \gamma)}=2 K_{+-}+K_{++}+K_{--}+\sum_{\mathrm{n}>2} \frac{n}{2} K_{\mathrm{n}}^{s} a^{n-2}
\end{gathered}
$$

with

$$
K_{i j}=4 \pi \int_{0}^{R} r^{2} \exp \left(-\frac{z_{i} z_{j}}{\left|z_{i} z_{j}\right|} \frac{2 q}{r}\right) d r
$$

This implies that at low concentrations the Bjerrum formulation is asymptotically exact, just as the DH limiting law is for instance, but with a much stronger efficiency in a wider range of application. Recalling now the results obtained formerly, (ref. 10), this also implies that in the short range region, $r<R$, the following expression

$$
\stackrel{\circ}{g}_{i j}=\gamma f_{ \pm}^{2}(R, c \gamma) \exp \left(-\frac{z_{i} z_{j}}{\left|z_{i} z_{j}\right|} \frac{2 q}{r}\right)
$$

is better than any of the above formulations since it can lead to an exact evaluation of the linear terms of the activity expansion, a performance that none of these can achieve. Similarly in the long-range region, the following (DHGX) equation

$$
\stackrel{g}{g}_{i j}=\gamma \exp \left(-\frac{z_{i} z_{j}}{\left|z_{i} z_{j}\right|} \frac{2 q}{r} e^{-\kappa \gamma^{1 / 2} r} \frac{e^{\kappa \gamma^{1 / 2} R}}{1+\kappa \gamma^{1 / 2} R}\right)+1-\gamma
$$

would constitute a definite improvement or even its truncated version (DHG). The latter would lead to a DH expression for $f_{ \pm}(R, c \gamma)$ in eq.(20), in conformity with Bjerrum's original proposal (ref 5). These analytical expressions are some simplified variant versions of those derived by Friedman (ref. 12), who matched the short- and long-range functions by imposing continuity up to first space-derivative, and by Grigo and Ebeling (ref. 13), who proved the necessity to achieve normalization when the distance $r$ tends to $\infty$.

\section{THE TRANSPORT EQUATIONS}

\section{6-1. Conductance}

\section{6-1-1. Symmetrical Electrolytes}

It has been shown elsewhere (ref. 6), that the use of the echo-equation(13) leads to the following formulation

with (ref. 14)

$$
\begin{gathered}
\Lambda=\gamma \Lambda_{0}\left(1+\frac{\Delta X_{c}^{L}}{X}+\frac{\Delta X_{h}}{X}+\frac{\Lambda_{e}}{\Lambda_{0}}\right) \\
\frac{1-\gamma}{\gamma}=-A_{+-c}
\end{gathered}
$$

$$
A_{+-}^{S}=-4 \pi \int_{0}^{R} r^{2} \stackrel{g}{g}_{+-}(r) d r
$$

Quite obviously the use of eq.(21) for $\hat{g}_{+-}(r)$ leads exactly to the Bjerrum definition of the fraction $\gamma$ of free ions. 


\section{6-1-2. Mixtures of electrolytes of any kinds}

The generalisation of the above result to the case of ionic mixtures has been carried out (ref. 15). The result reads

$$
\begin{gathered}
10^{3} \chi=\sum_{i} \gamma_{i} c_{i} \lambda_{0}^{i}\left(1+\frac{\Delta X_{i}^{L c}}{X}+\frac{\Delta X_{i}^{h}}{X}+\frac{\lambda_{e l}^{i}}{\lambda_{0}^{i}}\right)+\sum_{i} \sum_{k>i} c_{i}\left(1-\gamma_{i}\right) \lambda_{0}^{i k} H_{i k} \\
\frac{1-\gamma_{i}}{\gamma_{i}}=-\sum_{k \neq i} c_{k} \gamma_{k} K_{i k}^{\prime} \\
\lambda_{0}^{i k}=\left(z_{i}+z_{k}\right)^{2} \frac{\lambda_{0}^{k} \lambda_{0}^{i}}{\lambda_{0}^{k} z_{i}^{2}+\lambda_{0}^{i} z_{k}^{2}} .
\end{gathered}
$$

where $\chi$ is the specific conductance.

Again the interpretation of this result in term of ionic association is clear. Let us emphasize that $\lambda_{0}^{i k}$ identifies exactly with the ionic conductance of an entity composed of two ions $i$ and $k$ which would be tightly linked together. With this result, one obtains for the first time the mere kinetic definition of two associated ions without using that concept as a model at all in the theoretical derivation.

\section{6-2. Tracer diffusion}

Using the RPM, Turq (ref. 16), has derived an equation for tracer diffusion with the same method and the same approximations as Fuoss and Onsager for conductance. The result can be summarized as

$$
D^{1}=D_{0}^{1}\left(1+\frac{\Delta D_{T}^{1}(a, c)}{D_{0}^{1}}\right)
$$

The utilisation of the echo-equation(13) leads to the following generalization

$$
D^{1}=\gamma D_{0}^{1}\left(1+\frac{\Delta D_{T}^{1}(R, c \gamma)}{D_{0}^{1}}\right)+(1-\gamma) \frac{D_{0}^{1} D_{0}^{3}}{D_{0}^{1}+D_{0}^{3}}
$$

where the ions of type 3 are the ions of the non-labelled electrolyte which carry a charge opposite to that of the tracer ion 1.

The demonstration of eq.(25) is given with some details as an example in the appendix B. This result legitimizes the identical expression which Turq et al. found useful to introduce empirically, in order to explain quantitatively their experimental results in solvents of lower dielectric constant (ref. 17,18).

\section{6-3. Chemical diffusion}

For a binary symmetrical electrolyte, the condition of electroneutrality requires that both ions of the electrolyte diffuse with the same velocity

$$
\mathbf{v}^{i P}=\mathbf{v}^{j Q}=\mathbf{v}
$$

The origin of this equality in the set of equations of table 1 , is to be sought as the contribution of the non-zero $g^{\nabla i P}$ and $g_{j Q}^{\nabla i P}$ functions to the force $\mathrm{F}^{i P}$ in eq.(3). It is generally implicitly assumed that this first contribution has no concentration dependence. A consequence of the equality of the one-particle velocities is that the perturbation functions $g_{i j}^{\prime}$ given by eq.(13), are null and do not cause any relaxation effect. On the other hand, each ion induces, through its motion, a flow in the supporting medium which leads to an electrophoretic velocity. It is assumed in the evaluation of this effect that the $g_{i j}^{\nabla}$ functions contribute in a negligible way so that one needs only to take in consideration there, the equilibrium values $\stackrel{g}{i j}_{i j}$.

Once these assumptions are made, the evaluation of the chemical diffusion coefficient can be carried out according to the briefly summarized following procedure.

The driving force acting on an ion $i$ is

$$
\mathbf{k}_{i} \equiv \mathbf{F}^{i P}=\frac{\mathbf{v}}{\omega_{i}}\left(1-\frac{\Delta v_{i}}{v}\right)
$$

since it induces only that part of the total velocity which is not electrophoretic.

The electrophoretic velocity $\Delta v_{i} \equiv v e^{i P}$ must be evaluated from eq.(6) with the following specialization:

The external force $\mathbf{K}_{i}$ is null.

The internal forces $\mathbf{F}_{i P}^{l S}$ reduce to the driving forces $\mathbf{F}^{l S} \equiv \mathbf{k}_{l}$, since there are no relaxation contributions. 
The equivalence symbols give the correspondence with the notation used by Pikal (ref. 19).

Substituting eq.(26) in eq.(6) shows that each electrophoretic velocity turns out to follow the formulation

$$
\frac{\Delta v_{i}}{v}=\alpha_{i i}\left(1-\frac{\Delta v_{i}}{v}\right)+\alpha_{i j}\left(1-\frac{\Delta v_{j}}{v}\right)
$$

With these relations, one reaches a similar situation to that obtained with the echo effect on the relaxation contribution in conductance and tracer diffusion: Each electrophoretic contribution $\Delta v_{i}$ is a linear function of $\Delta v_{i}$ and $\Delta v_{j}$. This was already mentioned by Onsager and Fuoss in their original publication (cf. ref. 4, page 2765) but not used since their derivation concerned the limiting law only. On the other hand, Pikal rightly used this property and substituted the Onsager-Fuoss limiting laws for $\Delta v_{l} / v$ in the RHS of eq.(27).

The total driving force acting on the electrolyte is $k=\nu_{1} \mathbf{k}_{1}+\nu_{2} \mathbf{k}_{2}$. The thermodynamic diffusion coefficient is now defined by

$$
M=\frac{v}{k}=\frac{M_{0}}{1-\Delta M / M_{0}}
$$

where

$$
M_{0}=\nu_{1} / \omega_{1}+\nu_{2} / \omega_{2}
$$

and

$$
\Delta M=\frac{\nu_{1}}{\omega_{1}} \frac{\Delta v_{1}}{v}+\frac{\nu_{2}}{\omega_{2}} \frac{\Delta v_{2}}{v}
$$

The chemical diffusion coefficient $D$ which is the measured quantity proceeds from $M$ by

$$
D=R T \nu M\left(1+m \frac{d \ln \gamma_{ \pm}}{d m}\right)
$$

where $m$ is the molality and $\gamma_{ \pm}$the mean activity coefficient in the molal scale of the electrolyte of stechiometric coefficient $\nu=\nu_{1}+\nu_{2}$. This conversion takes into account the difference in the definitions of $M$, (diffusion along the chemical potential gradient), and of $D$, (diffusion along the concentration gradient).

Formulation(28) is equivalent to eq.(B4) obtained by application of the echo effect to self-diffusion. It is then possible to extract from Pikal's result for $\Delta M$ the function $\Delta M_{A}$ which contains the short-range anion-cation contribution

$$
\Delta M=\Delta M_{A}+\Delta M^{\prime}=\Delta M_{A}^{S}+\Delta M_{A}^{L}+\Delta M^{\prime}
$$

and to proceed to the same analytical transformation which led from eq.(B4) to eq.(B7). The result reads

$$
M=\gamma M_{0} /\left[1-\gamma\left(\frac{\Delta M_{A}^{L}}{M_{0}}+\frac{\Delta M^{\prime}}{M_{0}}\right)\right]+(1-\gamma)(1+B) M_{0} \times A^{\prime}
$$

with

$$
\begin{gathered}
A^{\prime}=M / M_{0}\left[1-\gamma\left(\frac{\Delta M_{A}^{L}}{M_{0}}+\frac{\Delta M^{\prime}}{M_{0}}\right)\right] \\
B=2 N_{A} F \nu_{1}\left|z_{1}\right| / 3 \pi \eta q \Lambda_{0} C \times 10^{-11} \\
\frac{1}{B} \frac{\Delta M_{A}^{S}}{M_{0}}=\frac{1-\gamma}{\gamma}=K_{A}^{\prime} f^{2}
\end{gathered}
$$

and

$$
f^{2}=\exp [-2 \kappa q /(1+\kappa a)]
$$

The result for $K_{A}^{\prime}$ practically identifies with the Bjerrum constant $K_{A}^{B}$ since

$$
\begin{aligned}
K_{A}^{B} & =K_{0}\left[Q\left(\frac{2 q}{a}\right)-Q\left(\frac{2 q}{R}\right)\right] \\
K_{A}^{\prime} & =K_{0}\left[Q^{\prime}\left(\frac{2 q}{a}\right)-Q^{\prime}\left(\frac{2 q}{R}\right)\right]
\end{aligned}
$$

where

$$
Q(x)=E_{p}(x)-\frac{e^{x}}{x}\left(1+\frac{1}{x}+\frac{2}{x^{2}}\right)
$$

whereas the function found by Pikal reads

$$
Q^{\prime}(x)=E_{p}(x)-\frac{e^{x}}{x}\left(1+\frac{1}{x}\right)
$$


The improvement of Pikal's result in eq.(30) over that of Turq for $\xi_{13}^{s}$ in eq.(B9) for the same quantity is due to the fact that Pikal made use of the DHX approximation whereas Turq used only the usual DH one for the equilibrium distribution functions $\stackrel{\circ}{i j}_{i j}$ (cf. section 5). Again, if these authors had used eq.(21), the exact formulation of the Bjerrum mass-action law given by eq.(B10) would have been obtained.This remark emphasizes the advantage of this new formulation. It should certainly improve the efficiency of the Pikal theoretical equation as was observed already for conductance and tracer diffusion. Finally it also shows that the counter part of using this reformulation is to substitute the cut-off length value $R$ to the characteristic distance $a$ in the leading function $\Delta M_{A}(a)$, a fact which was refuted by Pikal in his conclusions since, indeed, this is not explicitly obvious in the original formulation(28), just as it is not apparent in eq.(B4) for tracer diffusion as well as in the full echo-formulation of conductance.

A simplified version of eq.(29), obtained from considerations based on a chemical model of association, has already been used with success by Tanaka et al., to process numerically chemical diffusion coefficient data for 2-2 electrolytes in water (ref. 20).

\section{CONCLUSIONS}

In the brief survey given here of the excess transport properties of electrolyte solutions, we have shown how a formulation in agreement with the model of ionic association can be reached, without making any empirical use of that model, but rather by taking into account some subtle aspects of the fundamental equations as they result from basic statistical mechanical considerations. It was also shown that to obtain an optimal efficiency of this formulation, it was necessary to make use of a formulation for equilibrium pair distribution which explicitly takes into consideration some important results obtained from the Mayer activity expansion. This implies a space partition at a cut-off distance $R$. In the outer region $(r>R)$, the usual Debye truncated expansion of the new $\dot{g}_{+-}(c \gamma)$ function may be used, which implies that the concentration $c$ be replaced by $c \gamma$ and the RPM distance $a$ by $R$. In the inner region $(r<R)$, it is much more efficient to take explicitly into account the echo formulation and to make use of the new expression(21) for the equilibrium distribution function.

Numerical analysis shows that in the range $0.5 q>R>2 q$ no deviation is observed for the calculated quantity within a precision of $0.01 \%$. In addition to the net improvement observed when these results are applied to solutions of ions of higher charges and/or of solvents of lower dielectric constant, one sees that these results are not restricted solely to the RPM model,at least for the important contributions involving ions of opposite charges. Indeed all $U_{i j}$ functions with $z_{i} z_{j}<0$ appear explicitly in terms which identify with classical association constants.

One finally sees that a unified level of approximation is now reached in the analytical expressions for equilibrium and transport excess properties in the case of linear response irreversibility. The interesting result which predominates is that, in the concentration range in which these analytical solutions remain valid,

$$
\kappa q \gamma^{1 / 2}<0.5
$$

which is imposed by the somewhat restrictive long-range approximations still in use, all the specificity of the ionic interactions remain free to be fixed explicitly since they appear in the Boltzmann integrals of the various $U_{i j}$ functions which define the model.

ACKNOWLEDGEMENT, Dr. A. Pethybridge's kind assistance in editing this manuscript is gratefully appreciated.

\section{APPENDIX A}

The hydrodynamic tensor $\vec{\chi}$ is the sum of two tensors

$$
\overrightarrow{\vec{\chi}}\left(P, S, \omega_{i}, \omega_{1}\right)=\overrightarrow{\vec{\lambda}}(P, S)+\vec{\lambda}^{\prime}\left(P, S, \omega_{i}, \omega_{1}\right)
$$

with a long-range contribution

$$
\left.\vec{\lambda}(P, S)=(1 / 8 \pi \eta r) \mid \vec{\delta}+\vec{r} r / r^{2}\right)
$$

commonly called the Oseen tensor and a short-range contribution (ref. 21), which so far has always been neglected

$$
\overrightarrow{\lambda^{\prime}}\left(P, S, \omega_{i}, \omega_{1}\right)=\left(1 / 8 \pi \eta r^{3}\right)\left(\omega_{i}^{-2}+\omega_{1}^{-2}\right)\left(\vec{\delta} / 3-\vec{r} r / r^{2}\right)
$$

where $\vec{\delta}$ is the unit tensor and $\vec{r} r$ the dyadic operator which, by definition, performs the following vectorial operation on a vector $\mathbf{V}$

$$
\vec{r} \cdot \mathbf{V}=\mathbf{r}(\mathbf{r} \cdot \mathbf{V})
$$




\section{APPENDIX B}

Let us consider the case of a ternary electrolyte where the labeled ions 1 , which are at infinitesimal concentration, are of the same chemical type as ions 2 and where $z_{2}=-z_{3}$. In order to satisfy the electroneutrality condition, the balance of ionic flows of ions 1 and 2 is such that the velocity of ions 2 is negligible compared to that of ions 1 . The assumption that ions 1 and 2 cannot be distinguished, except for their nuclear instability, leads to the conclusion that the counterions 3 effectively behave as in the equilibrium state, so that their velocity is null.

As a consequence, according to eq.(3), the perturbations in the distribution functions of ions 2 and 3 around the tracer ion 1 reduce to

$$
g_{1 k}^{\prime}(\mathbf{r})=-Y_{1 k}(r) \cos \theta \frac{\mathbf{v}^{1 P} \nabla x}{k_{B} T\left(\omega_{1}+\omega_{k}\right)}
$$

Substituting

$$
\omega_{i}=D_{0}^{i} / k_{B} T
$$

and

$$
\mathbf{v}^{1 P}=-D^{1} \nabla \ln ^{1 P}=-D^{1} \nabla \operatorname{lnc} c_{1}
$$

in eq.(B1), it comes

$$
g_{1 k}^{\prime}=-Y_{1 k}(r) \cos \theta \frac{D_{0}^{1}}{D_{0}^{1}+D_{0}^{k}}\left(1+\Delta D_{1} / D_{0}^{1}\right) \frac{\partial}{\partial x} \ln c_{1}
$$

Another consequence is that the tracer ion will experience practically no electrophoretic velocity. This is confirmed by an inspection of eq.(6) specialized to the case $i=1$ and $l=2,3$. Indeed, in diffusion the external forces $\mathbf{K}_{i}$ are null and in tracer diffusion both contributions of $\mathrm{F}_{1 P}^{2 S}$ and $\mathrm{F}_{1 P}^{3 S}$ to $\mathrm{v} e^{1 P}$ are completely negligible compared to that of $\mathrm{F}^{1 P}$ to $\mathrm{v}^{1 P}$ in eq.(1), as indicated by eq.(4) for these two quantities.

The problem of tracer diffusion thus reduces to the evaluation of the relaxation force $\mathbf{F}^{1 P}$ by eq. $(6)$ in which $l=2,3$. The result can be expressed by

$$
\mathbf{F}^{1 P}=\mathbf{k}_{1}+\Delta \mathbf{F}^{1 P}
$$

where $\mathbf{k}_{1}$ is the driving force

$$
\mathbf{k}_{1} \nabla x=k_{1}=-k_{B} T \frac{\partial}{\partial x} \ln c_{1} .
$$

The substitution of eq.(B3) in eq.(1) leads to

$$
\frac{v^{1 P}}{k_{1}}=\omega_{1}\left(1+\frac{\Delta F^{1 P}}{k_{1}}\right)
$$

After appropriate unit conversion, that is after deviding by $k_{B} T$, one reaches

$$
D_{1}=D_{0}^{1}\left(1+\frac{\Delta D_{1}}{D_{0}^{1}}\right) \text {. }
$$

In the usual approximation, these calculations are performed in neglecting the excess term $\Delta D_{1} / D_{0}^{1}$ in eq.(B2). The result will then be denoted

$$
D_{I 1}=D_{0}^{1}\left(1+\frac{\Delta D_{I 1}}{D_{0}^{1}}\right)
$$

and it is easy to show that the exact formulation which is self-consistent with eq.(B2) is very simply given by

$$
D_{1}=\frac{D_{0}^{1}}{1-\Delta D_{I 1} / D_{0}^{1}}
$$

All the integrals involved in eq.(3) can be devided into short-range and long-range contributions. Let us define

$$
\frac{\Delta D_{1 l}^{S}}{D_{0}^{1}}=-\frac{n_{l}}{k_{1}} \int_{Q \in \Omega} \nabla_{P} U(1 P, l Q) g_{1 P}^{\prime \prime Q} d V_{Q}
$$

and

$$
\frac{\Delta D_{1 l}^{L}}{D_{0}^{1}}=-\frac{n_{l}}{k_{1}} \int_{Q \ni \cap} \nabla_{P} U(1 P, l Q) g_{1 P}^{\prime Q Q} d V_{Q}
$$

where $\Omega$ is a sphere of arbitrary radius $R$ centered on point $P$. Their sum represents the part of $\Delta D_{1}$ which is due to the interactions of ions $l \neq 1$ on the tracer ions. 
One observes that the classical derivation is in fact the linearization (after expansion) of the echo formulation. This conclusion is the same as that reached for conductance. One might thus use directly the echo-formula(B4), but this is however not necessary, nor even recommanded, since in fact both $D_{I 12}$ and $D_{I 13}$ are not known with enough precision. Much of the numerical "improvement" which the echo-formulation should bring is lost by the fact that the long-range parts $D_{I 12}^{L}$ and $D_{I 13}^{L}$ are not known with enough precision. However, this is not the case of the short-range contribution $\Delta D_{I 13}^{S}$, of which the linear contribution with concentration may be evaluated with precision so that the improvement may become very important.

As a consequence of this remark, one should restrict the application of the echo-formulation only to $\Delta D_{13}^{S}$ as defined above in eq.(B5). One may rewrite the self-diffusion equation as

$$
D_{1}=D_{0}^{1} /\left(1-\frac{\Delta D_{I 13}^{S}}{D_{0}^{1}}-\frac{\Delta D_{I 12}^{S}}{D_{0}^{1}}-\frac{\Delta D_{I 1}^{L}}{D_{0}^{1}}\right)
$$

where

$$
\frac{\Delta D_{I 1}^{L}}{D_{0}^{1}}=\frac{\Delta D_{I 12}^{L}}{D_{0}^{1}}+\frac{\Delta D_{I 13}^{L}}{D_{0}^{1}}
$$

Due to the opposite charges of ions 1 and 3, the perturbation $g_{13}^{\prime}$ can become numerically important at short distances for ions of higher charge numbers or solvents of lower dielectric constant. An analysis of eqs.(B2,B5) shows that the short rangecontributions can be expressed by

$$
\frac{\Delta D_{I 1 k}^{\varsigma}}{D_{0}^{1}}=\xi_{1 k}^{\varsigma} \frac{D_{0}^{1}}{D_{0}^{1}+D_{0}^{3}}
$$

with

$$
\xi_{1 k}^{\mathrm{s}}=-\frac{n_{k}}{k_{B} T} \int_{R \in \Omega} \nabla_{\Gamma} U(1 P, k R) Y_{1 k}(r) \cos \theta d V_{R}
$$

Eq.(B4) can now be rewritten as

$$
D_{1}=D_{0}^{1} /\left(1-\xi_{13}^{S} \frac{D_{0}^{1}}{D_{0}^{1}+D_{0}^{3}}-\xi_{12}^{\mathrm{S}} \frac{D_{0}^{1}}{D_{0}^{1}+D_{0}^{2}}-\frac{\Delta D_{I 1}^{L}}{D_{0}^{1}}\right)
$$

or

$$
D_{1}=D_{0}^{1} /\left(1-\xi_{13}^{S}+\xi_{13}^{S} \frac{D_{0}^{3}}{D_{0}^{1}+D_{0}^{3}}-\xi_{12}^{S} \frac{D_{0}^{1}}{D_{0}^{1}+D_{0}^{2}}-\frac{\Delta D_{I 1}^{L}}{D_{0}^{1}}\right)
$$

Factorizing now the quantity

$$
\gamma=\frac{1}{1-\xi_{13}^{\varsigma}}
$$

it becomes

$$
D_{1}=\gamma D_{0}^{1} /\left[1-\gamma\left(\frac{\Delta D_{I 1}^{L}}{D_{0}^{1}}+\xi_{12}^{\varsigma} \frac{D_{0}^{1}}{D_{0}^{1}+D_{0}^{2}}\right)-(1-\gamma) \frac{D_{0}^{3}}{D_{0}^{1}+D_{0}^{3}}\right]
$$

and finally

$$
D_{1}=\gamma D_{0}^{1} /\left[1-\gamma\left(\frac{\Delta D_{I 1}^{L}}{D_{0}^{1}}+\xi_{12}^{\xi} \frac{D_{0}^{1}}{D_{0}^{1}+D_{0}^{2}}\right)\right]+(1-\gamma) \frac{D_{0}^{1} D_{0}^{3}}{D_{0}^{1}+D_{0}^{3}} A
$$

with

$$
A=D_{1} / D_{0}^{1}\left[1-\gamma\left(\frac{\Delta D_{I 1}^{L}}{D_{0}^{1}}+\xi_{12}^{\varsigma} \frac{D_{0}^{1}}{D_{0}^{1}+D_{0}^{2}}\right)\right]
$$

and

$$
\frac{1-\gamma}{\gamma}=-\xi_{13}^{\mathrm{s}}
$$

Some remarks are appropriate at this point. So far, the system of equations(B7-B9) is identical to the simple echo-formulation (B4), and the transformation may seem a useless complication. However, if one improves the evaluation of $\xi_{13}^{S}$ which concerns the analytical treatment of the short-range anion-cation interactions (between ions of type 1 and 3), it becomes substantially more efficient than any former derivation. Indeed, when the coulombic attraction becomes large between the ions of opposite charges 1 and 3, one observes that $\gamma$ is a number which varies from 0 to 1 , since $\xi_{13}^{\mathcal{S}}$ can vary from 0 to $-\infty$. The second term in the RHS of eq.(B7) can easily be interpreted as the contribution to the diffusion of the fraction $1-\gamma$ of the ions of type 1 which are coupled with ions of type 3 to form pairs of ions which would diffuse as a single kinetic entity. The first term in eq.(B7) corresponds to the fraction $\gamma$ of the ion of type 1 which are free (as a kinetic entity). Since, in opposition, the term $\xi_{12}^{\mathrm{S}} \frac{D_{0}^{1}}{D_{0}^{1}+D_{0}^{2}}$ is always numerically very small as it corresponds to short-range configurations of ions of same algebraic sign, the 
diffusion coefficient of the free ions is, through the term $\frac{\Delta D_{t}^{x}}{D_{0}^{1}}$, practically a function of $\mathrm{R}$ only, the arbitrary cut-off distance which is chosen in the space partition used in eq.(B5). This is in conformity with the initial proposition of Bjerrum of 1923, who empirically used a priori the chemical model of ionic association to correct the Debye-Huckel activity coefficient law.

Coming back to eq.(B9) it is easy to show that the quantity $\xi_{13}^{S}$ formally identifies with the homologous conductance term $A_{+-}^{S}$ of eq.(22) so that if use is made of the approximation proposed for $\stackrel{\mathscr{g}}{+}_{+-}^{S}$ given by eq.(21), $\gamma$ is then given by the complete mass-action law

$$
\frac{1-\gamma}{\gamma^{2} c f_{ \pm}^{2}(R, c \gamma)}=2 K_{+-}
$$

One should be cautious though in making use of eq.(B7) and retain in it only those contributions which can be considered as exact.

This is the case of the coefficients $\gamma$ and $(1-\gamma)$ which are factors in both of the terms in the RHS of eq.(B7). Indeed, their chief contribution, which is linear in concentration, is proved to be analytically known exactly. This is not the case of the contribution of $\gamma$-term in the denominator of the first term, nor of $A$ in the second term of the RHS of eq.(B7). There are strong indications that the long-range functions which they contain are not sufficiently evaluated to be adequately corrected that way, that is to match the potential improvement which is offered. Waiting for more progress in that field it is better to fix $\gamma=1$ and $A=1$ in those terms, that is, to use them in the usual linear expansion approximation.

As a result, the best that can be proposed for application of the echo-effect at this moment is

$$
D_{1}=\gamma D_{0}^{1}\left(1+\frac{\Delta D_{I 1}^{L}}{D_{0}^{1}}+\xi_{12}^{\varsigma} \frac{D_{0}^{1}}{D_{0}^{1}+D_{0}^{2}}\right)+(1-\gamma) \frac{D_{0}^{1} D_{0}^{3}}{D_{0}^{1}+D_{0}^{3}}
$$

which already constitutes a significant improvement when $\gamma$ is calculated with eq.(B10).

If now one recalls that the $\xi_{12}^{\mathcal{S}}$ contribution is always numerically very small and can be dropped, one obtains a full legitimation of the equation(25) which also results more directly from a strict application of the Bjerrum concept of association.

\section{REFERENCES}

1. P. Debye and E. Huckel, Physik. Zeit., 24, 185 (1923).

2. P. Debye and E. Huckel, Physik. Zeit., 24, 305 (1923).

3. P. Debye and H. Falkenhagen, Physik. Zeit., 29, 401 (1928).

4. L. Onsager and R.M. Fuoss, J. Phys. Chem., 36, 2689 (1932).

5. N. Bjerrum, K. Dan. Videnskab. Selsk. Mat. Fys. Skr., 7,1 (1926); see also 'Niels Bjerrum's Selected papers' (English translation), Einer Munksgaard Pub., Copenhagen, 1949; and 'J.W. Bronsted and Niels Bjerrum - A Centenary Report', edited by Thor A. Bak, University of Copenhagen, 1984.

6. J.C. Justice, J. Solution Chem., 7, 859 (1978).

7. W. Ebeling, Wiss.Z. Univ. Rostock Math. Natur Wiss. 14, 271 (1965); see also H. Falkenhagen, W. Ebeling and W.D. Kraeft, in 'Ionic Interactions' Vol. 1, Chapter 2, edited by S. Petrucci, Academic Press, 1971; and H. Falkenhegen, W. Ebeling and H.G. Hertz, in 'Theorie der Elektrolyte' - S. Hirzel Verlag, Leipzig, 1971.

8. J.C. Justice, in 'Comprehensive Treatise of Electrochemistry', Vol. 5, Chapter 3, edited by B.E. Conway, J. O'M. Bockris and E. Yeager, Plenum, 1983.

9. M.C. Justice and J.C. Justice, J. Solution Chem., 5, 543 (1976).

10. M.C. Justice and J.C. Justice, J. Solution Chem., 6, 819 (1977).

11. J.C. Justice, to be submitted to J. Solution Chem.

12. H. L. Friedman, J. Solution Chem., 9, 371 (1980).

13. M. Grigo and W. Ebeling, J. Solution Chem., 13, 321 (1984).

14. J.C. Justice and W. Ebeling, J. Solution Chem., 8, 809 (1979).

15. C. Micheletti and J.C. Justice to be submitted to J. Solution Chem.

16. P. Turq, Chem. Phys. Letters, 15, 579 (1972).

17. P. Turq, D. Ilzycer and M. Chemla, J. Chim. Phys., 2, 233 (1974).

18. H. Latrous,P. Turq and M. Chemla, J. Chim. Phys., 11, 1650 (1972).

19. M. J. Pikal, J. Phys. Chem., 75, 663 (1971).

20. K. Tanaka, T. Hashitani and R. Tamamushi, Report of the Institute of Physical and Chemical Research, 51, 153 (1975) at Hirosawa, Wako-Shi, Saitama, 351 Japan.

21. U. Felderhof, Physika, 89A, 373 (1979). 